typeset using JPSJ.sty $<$ ver.1.0b $>$

\title{
Magnetization Process of Nanoscale Iron Cluster
}

\author{
Hiroki NAKANO and Seiji MiYashita \\ Department of Applied Physics, School of Engineering, University of Tokyo, \\ Hongo 7-3-1, Bunkyo-ku, Tokyo 113-8656
}

(Received November 14, 2018)

\begin{abstract}
Low-temperature magnetization process of the nanoscale iron cluster in linearly sweeped fields is investigated by a numerical analysis of time-dependent Schrödinger equation and the quantum master equation. We introduce an effective basis method extracting important states, by which we can obtain the magnetization process effectively. We investigate the structure of the field derivative of the magnetization. We find out that the antisymmetric interaction determined from the lattice structure reproduces well the experimental results of the iron magnets and that this interaction plays an important role in the iron cluster. Deviations from the adiabatic process are also studied. In the fast sweeping case, our calculations indicate that the nonadiabatic transition dominantly occurs at the level crossing for the lowest field. In slow sweeping case, due to the influence of the thermal environment to the spin system, the field derivative of the magnetization shows an asymmetric behavior, the magnetic Föhn effect, which explains the substructure of the experimental results in the pulsed field.
\end{abstract}

KEYWORDS: Nanoscale magnet, magnetization process, time-dependent Schrödinger equation, quantum master equation, Lanczos diagonalization, Runge-Kutta method, nonadiabatic transition

\section{$\S 1$. Introduction}

Nanoscale magnets have attracted much interest from the point of view of their quantum dynamics.1. As such magnetic materials, $\mathrm{Fe}_{n}(n=6,10,12,18)$ have been synthesized. In these compounds, each iron ion $\mathrm{Fe}^{3+}$ plays a role of an $S=5 / 2$ spin and forms a ringstructure molecule. Each of these compounds reveals a step-like magnetization process $M(H)$ at low temperatures. These steps appear due to the fact that the energy levels are discrete, which is one aspect of the quantum nature. First, let our attention be focused on the magpetizations differentiated by the magnetic field, $\mathrm{d} M / \mathrm{d} H$ 2) At magnetic fields where the magnetization grows rapidly, $\mathrm{d} M / \mathrm{d} H$ shows peaks with various heights. In two of the compounds $\left[\mathrm{M} \mathrm{Fe} 6\left(\mathrm{OCH}_{3}\right)_{1}(\mathrm{dbm})_{6}\right]^{+}$ $\left.(\mathrm{M}=\mathrm{Na}, \mathrm{Li})^{3}\right)$ and $\left[\mathrm{Fe}(\mathrm{OMe})_{2}\left(\mathrm{O}_{2} \mathrm{CCH}_{2} \mathrm{Cl}\right)\right]_{10}, 4$, which are abbreviated by $\mathrm{Fe}_{6}$ and $\mathrm{Fe}_{10}$ respectively, the peak at the lowest magnetic field has the largest height; the heights of the peaks get smaller gradually at higher fields. How does the difference of the peak heights appear?

The main structure of the magnetization process is determined by the Heisenberg-like exchange interactions between the iron ions, where the $z$-component of the magnetization $M_{z}$ is a good quantum number. Eigenstates of various values of $M_{z}$ show many crossings in the energy-level structure as function of the magnetic field $H$. The ground state is given by the lowest eigenstate at the given field, which changes piecewise. The typical case of antiferromangetc interactions is shown schematically in Fig. ??. The singlet ground state, which is nonmagnetic, is realized at $H=0$. At $H=H_{1}$ and $H_{2}$, the ground state changes, where the magnetization of the ground state increases. If a system includes only the Heisenberg-like exchange interactions, $\mathrm{d} M / \mathrm{d} H$ re- veals the $\delta$ function at the crossing. In experiments, however, they have finite peaks. There are two possible origins for the finite heights. One is an effect due to finite temperature. The other is an effect due to some quantum-mechanical operators. Let us consider the case of the thermal effect. At low temperatures, only the two eigenstates concerning the crossing are relevant. Main contribution in the partition function consists of the Boltzmann weights of these states, and the thermal behavior is characterized by the energy difference between them. This effect causes almost equivalent peaks at all the crossings in $\mathrm{d} M / \mathrm{d} H$, which is not the case in the experiments. Therefore, it seems that the origin of the difference between the peak heights is due to some operators which do not commute with $M_{z}$. What kind of operator does induce such a transition? In this paper, we investigate such interactions. We consider the lattice structure of the iron clusters and find the existence of the antisymmetric interaction, namely the Dzyaloshinsky-Moriya interaction. This interaction which does not commute with $M_{z}$ is found to reproduce well the feature observed experimentally in the magnetization process of the iron clusters.

It is known that when the parameter is swept crossing the transition point, the system shows the nonadiabatic transition. 5 , The transition rate depends on the sweeping speed of the field. In particular, the present case belongs to the so-called Landau-ZenerStükelberg (LZS) mechanism. Tunneling phenomena of the metastable magnetization of the Ising-type model werestudied in the viewpoint of the nonadiabatic transition.8) Here we treat a model for the nanoscale magnets and clarify how effects of the nonadiabatic transition appear.

The peaks in $\mathrm{d} M / \mathrm{d} H$ of $\mathrm{Fe}_{6}$ and $\mathrm{Fe}_{10}$ are known to 
be asymmetric in higher- and lower-field regions with respect to the center of each peak. In the receding side of the pulsed field, the slope of the peak in $\mathrm{d} M / \mathrm{d} H$ is gentle in comparison with that of the approaching side. In the extreme case, a satellite peak is accompanied in the receding side. Similar behavior of hysteresis in the magnetization process is known in $[\mathrm{Fe}(\text { salen }) \mathrm{Cl}]_{2}$ (sp-called $\left.\mathrm{Fe}_{2}\right)$ and $\mathrm{K}_{6}\left[\mathrm{~V}_{15} \mathrm{As}_{6} \mathrm{O}_{42}\left(\mathrm{H}_{2} \mathrm{O}\right)\right]$ (so-called $\left.\mathrm{V}_{15}\right)$.10) The behavior of the vanadium compound was investigated as influence of the thermal environment which is called magnetic Föhn effect 11) In this work, we investigate the magnetization process of $\mathrm{Fe}_{6}$ with the effect of environment using the quantum master equation. We find out that the asymmetry of the peaks of the iron clusters can be understood as an effect from the environment.

This paper is organized as follows. The next section is devoted to the model Hamiltonian and the calculation method. The antisymmetric interaction in the model is introduced, which explains the main structure of the peaks in $\mathrm{d} M / \mathrm{d} H$. As to the method, we introduce an effective basis method in a dynamical approach in the Schrödinger equation and also in the quantum master equation. In $\S 3$ the magnetization process at zero temperature is discussed. In $\S 4$ the magnetization process at finite temperatures is investigated. Concluding remarks are given in $\S 5$.

\section{§2. Hamiltonian and Method}

\subsection{Spin Hamiltonian}

We consider a system that has a large interaction of Heisenberg type between two neighboring spins. In addition, there presumably exists other anisotropic interactions determined from the lattice structure of the compound even though they are smaller than the Heisenbergtype one. Let us consider the lattice structure of the iron clusters. In both $\mathrm{Fe}_{6}$ and $\mathrm{Fe}_{10}$, two neighboring iron ions have a mirror symmetry with respect to the plane perpendicular to the line connecting these iron ions. In this case, it is known that the Dzyaloshinsky-Moriya (DM) interactions

$$
\mathcal{H}_{\mathrm{DM}}=\sum_{j=1}^{N_{\mathrm{s}}} \boldsymbol{D}_{j} \cdot\left[\boldsymbol{S}_{j} \times \boldsymbol{S}_{j+1}\right],
$$

may be present, and that $\boldsymbol{D}_{j}$ is parallel to the mirror plane. Here, $N_{\mathrm{s}}$ denotes the number of sites and $\boldsymbol{S}_{j}$ denotes an $S=5 / 2$ spin operator at site $j$. The system is periodic, i.e., $\boldsymbol{S}_{N_{\mathrm{s}}+1}=\boldsymbol{S}_{1}$. It is known that, in every two neighboring pairs, branches of atomic bonds are alternating. Therefore, $\boldsymbol{D}_{j}$ and $\boldsymbol{D}_{j+1}$ have opposite directions. In this work, we treat the regular hexagon $\left(N_{\mathrm{s}}=6\right)$ with alternating $\boldsymbol{D}_{j}$

$$
\boldsymbol{D}_{j}=(-1)^{j}(D, 0,0) \text {, }
$$

as shown in Fig. ??. Thus, as a candidate model for the system of $\mathrm{Fe}_{6}$ and $\mathrm{Fe}_{10}$, we examine the Hamiltonian

$$
\begin{gathered}
\mathcal{H}_{\mathrm{sys}}=\mathcal{H}_{0}+\mathcal{H}_{\mathrm{DM}} \\
\mathcal{H}_{0}=\sum_{j=1}^{N_{\mathrm{s}}}\left[J \boldsymbol{S}_{j} \cdot \boldsymbol{S}_{j+1}-h(t) S_{j}^{z}\right]
\end{gathered}
$$

where $J$ is an amplitude of antiferromagnetic exchange interaction, and $h(t)$ is the external magnetic field. Here we consider the case of linear sweeping, $h(t)=c t$. In this work we examine the case where $D$ is much smaller than $|J|$. A main structure of energy is determined by $\mathcal{H}_{0}$ as a function of the field, as shown schematically in Fig. ??. Because $J \gg D$, the effect of $\mathcal{H}_{\mathrm{DM}}$ appears only in the vicinity of a level crossing, and causes the avoided level crossing structure.

\subsection{Calculation method}

Let us obtain the magnetization process. In this paper, we introduce a dynamical approach to obtain the magnetization process. It is possible in principle to calculate the adiabatic magnetization process $M(h)$ of $\mathcal{H}_{\text {sys }}$ by diagonalization. However, in order to obtain smooth curves, very fine steps in the field are necessary, which requires a large number of diagonalizations. Moreover this dynamical approach provides information on the dynamical processes which cannot be obtained by diagonalization.

To obtain the magnetization process in the ground state $(T=0)$, we solve the time-dependent Schrödinger equation

$$
\mathrm{i} \hbar \frac{\mathrm{d}}{\mathrm{d} t}|\psi\rangle=\mathcal{H}_{\text {sys }}|\psi\rangle,
$$

numerically. This equation is made dimensionless by the scaling $\tilde{t}=J t / \hbar$. We take $J$ as an energy unit; $\tilde{h}=$ $h / J$ and $\tilde{D}=D / J$ are independent parameters in the Schrödinger equation. The external magnetic field $\tilde{h}$ is increased linearly from zero, which corresponds to the pulsed field in experiments. In particular, although the pulse velocity has a sinusoidal shape in experiments, here we approximate it by a constant velocity. As far as the velocity is small, we can obtain the adiabatic process in both cases. The antiferromagnetic ground state for $h=0$ is taken as an initial state.

To treat the thermal environment, we have to consider the effect of environment, where the total Hamiltonian is given by

$$
\mathcal{H}_{\text {tot }}=\mathcal{H}_{\text {sys }}+\mathcal{H}_{\text {int }}+\mathcal{H}_{\text {bath }}
$$

where $\mathcal{H}_{\text {bath }}$ denotes a Hamiltonian of the heat bath. $\mathcal{H}_{\text {int }}$ denotes a term of interaction, which plays a role of interface exchanging between the heat bath and the spin system. We take the heat bath as the free boson system, namely, $\mathcal{H}_{\text {bath }}=\sum_{\alpha} \hbar \omega_{\alpha}\left(b_{\alpha}^{\dagger} b_{\alpha}+1 / 2\right) . \quad \mathcal{H}_{\text {int }}$ is taken as $\mathcal{H}_{\text {int }}=\lambda \sqrt{\hbar} \sum_{\alpha}\left(b_{\alpha}^{\dagger}+b_{\alpha}\right) X$, where $X$ is an operator in the spin system. Here, $\left\langle\phi_{M}|X| \phi_{M^{\prime}}\right\rangle$ is taken as a unit when $\left|M-M^{\prime}\right|=1$ and vanishes otherwise. Under the condition of small coupling $\lambda$, the quantum master equation for the density matrix

$$
\frac{d \rho(t)}{d t}=\frac{1}{\mathrm{i} \hbar}\left[\mathcal{H}_{\mathrm{sys}}, \rho(t)\right]-\Gamma \rho(t),
$$

has been derived.12) Here,

$$
\Gamma \rho(t)=\left\{[X, R \rho(t)]+[X, R \rho(t)]^{\dagger}\right\} \lambda^{2} \pi / \hbar,
$$




$$
\begin{gathered}
\langle k|R| n\rangle=\frac{1}{\hbar} X_{k, n} \Phi\left(\frac{E_{k}-E_{n}}{\hbar}\right), \\
\Phi(\omega)=\frac{I(\omega)-I(-\omega)}{\mathrm{e}^{\beta \omega}-1},
\end{gathered}
$$

where $\beta$ is the inverse of temperature, namely $k_{\mathrm{B}} T=$ $1 / \beta$. Here, $|k\rangle$ and $|n\rangle$ represent eigenstates for $\mathcal{H}_{\text {sys }}$ with energy eigenvalues $E_{k}$ and $E_{n}$, respectively. If we assume that a main contribution of the heat bath comes from the lattice, the bosons in the environment are considered to be phonons. Then, the spectral density $I(\omega)$ is given by $I_{0} \omega^{2} \theta(\omega)$ where $\theta(\omega)$ is the step function. We solve the quantum master equation (2.7) to obtain the magnetization process at finite temperatures.

\subsection{Effective basis method}

Since the number of states $N_{\mathrm{D}}=(2 S+1)^{N_{\mathrm{s}}}=46656$ is large, solving the Schrödinger equation (2.5) using all the states in the system is difficult, in particular for long times. The calculation of the quantum master equation (2.7) is even more difficult because the number of elements of the density matrix is $N_{\mathrm{D}}^{2}$. Therefore, some approximation method which extracts important states of the system is required.

Now we introduce an approximate method for this purpose. First note here that many eigenstates are irrelevant to study the magnetization growth with the increasing field in the ground state through the quantum transitions of the present interests. In spite of this fact, the irrelevant states and important states are treated equivalently in full-size calculations, and this is inefficient. Now let us consider a method to extract the important states which contribute to the quantum transitions.

Here, the following procedure is performed, which we call an effective basis method. At first, one obtains the ground state $\left|\psi_{\mathrm{G}}\right\rangle$ of $\mathcal{H}_{\text {sys }}$ for a field $\tilde{h}(\tilde{t})$ at a time $\tilde{t}$ (initially, $\tilde{t}=0$ ) by the Lanczos diagonalization in the basis taking the $z$ direction as the quantization axis. Note that for the present system with dimension $N_{\mathrm{D}}=46656$, this is still possible. From $\left|\psi_{\mathrm{G}}\right\rangle$, one extracts $\left|\phi_{M_{z}}\right\rangle=\left|\tilde{\phi}_{M_{z}}\right\rangle / \sqrt{\left\langle\tilde{\phi}_{M_{z}} \mid \tilde{\phi}_{M_{z}}\right\rangle}$, where

$$
\left|\tilde{\phi}_{M_{z}}\right\rangle=\sum_{k}\left|\xi_{k}\right\rangle\left\langle\xi_{k} \mid \psi_{\mathrm{G}}\right\rangle
$$

where $\left|\xi_{k}\right\rangle$ denotes the $k$-th basis state with the magnetization $M^{z}$, i.e., $S_{z}^{\text {tot }}\left|\xi_{k}\right\rangle=M^{z}\left|\xi_{k}\right\rangle$. Here, $S_{z}^{\text {tot }}$ is defined by $\sum_{j=1}^{N_{\mathrm{s}}} S_{j}^{z}$. Note that $M_{z}$ is an integer ranging between zero and the saturation value, which is equal to $15\left(=\frac{5}{2} \times 6\right)$ for the present case. The new state $\left|\phi_{M_{z}}\right\rangle$ is a representative of the states of $M_{z}$. We have checked that the set of states $\left\{\left|\phi_{M_{z}}\right\rangle\right\}$ represents well the behavior of $\mathcal{H}_{\text {sys }}$ at low energy. Using this set of states, one constructs a small matrix

$\tilde{\mathcal{H}}_{\text {sys }}=$

$\left[\begin{array}{cccc}\left\langle\phi_{0}\left|\mathcal{H}_{\text {sys }}\right| \phi_{0}\right\rangle & \left\langle\phi_{0}\left|\mathcal{H}_{\text {sys }}\right| \phi_{1}\right\rangle & \cdots & \left\langle\phi_{0}\left|\mathcal{H}_{\text {sys }}\right| \phi_{15}\right\rangle \\ \left\langle\phi_{1}\left|\mathcal{H}_{\text {sys }}\right| \phi_{0}\right\rangle & \left\langle\phi_{1}\left|\mathcal{H}_{\text {sys }}\right| \phi_{1}\right\rangle & \cdots & \left\langle\phi_{1}\left|\mathcal{H}_{\text {sys }}\right| \phi_{15}\right\rangle \\ \vdots & \vdots & \ddots & \vdots \\ \left\langle\phi_{15}\left|\mathcal{H}_{\text {sys }}\right| \phi_{0}\right\rangle & \left\langle\phi_{15}\left|\mathcal{H}_{\text {sys }}\right| \phi_{1}\right\rangle & \cdots & \left\langle\phi_{15}\left|\mathcal{H}_{\text {sys }}\right| \phi_{15}\right\rangle\end{array}\right]$

Here, we take only the ground state to generate the effective basis. In this paper, we find that even in the present basis, we obtain good results. However, we can improve the approximation by adding more basis states extracted from a few low-energy states.

In this work, we perform Runge-Kutta calculations for the small matrix $\tilde{\mathcal{H}}_{\text {sys }}$ instead of the full-size matrix $\mathcal{H}_{\text {sys }}$. In the Runge-Kutta calculations, the normalization of the wave function deviates easily from unity. We have checked it carefully and used a small enough time step. The initial condition is $\left|\phi_{0}\right\rangle$. To keep the quality of the approximation the same, we update a set $\left\{\left|\phi_{M_{z}}\right\rangle\right\}$ by the above mentioned method, i.e. (2.11), after time evolution by $\mathcal{H}$ for a while. It is found that $\left\{\left|\phi_{M_{z}}\right\rangle\right\}$ gives a good approximation if it is updated as often as the level crosses. We have verified that no significant differences appear when the frequency of the update is varied. When the sweeping speed is small and the system behaves adiabatically, the states with the lowest and the second lowest energies are important. The method described here gives the magnetization process $M(h)$ as the adiabatic process very effectively. Actually, this dynamical method is a natural way corresponding to experiments of pulsed magnetic field.

As we mentioned, the present method provides information of nonadiabatic process for fast sweepings, that cannot be obtained by a static Lanczos diagonalization only. To demonstrate the validity of the above approximation for the nonadiabatic transition, we have performed the calculation of the Schrödinger equation for $S=1$ for $\tilde{D}=0.01$. In this case, the first avoided level crossing has an energy gap $\Delta E / J \sim 0.0196$. According to the LZS mechanism, the transition rate at an avoided crossing is given by

$$
P=1-\exp \left(-\frac{\pi(\Delta E / J)^{2}}{2\left|M_{z}-M_{z}^{\prime}\right| \tilde{h}_{\max } / \tilde{t}_{\max }}\right),
$$

when the magnetic field is linearly sweeped from zero to $\tilde{h}_{\max }$ during a time of $\tilde{t}_{\max }$. For the above energy gap, $\tilde{h}_{\max }=6, \tilde{t}_{\max }=3000, M_{z}=1$ and $M_{z}^{\prime}=0$, the transition rate $P$ given by (2.13) is 0.26 . After the corresponding level crossing, the present approximation gives for this rate 0.26 . This agreement indicates the validity of the above approximation. For the quantum master equation, we use the same effective basis. We have also checked the agreement of a result from the quantum master equation for a full-size Hamiltonian with a small dimension and that from the quantum master equation for the Hamiltonian with its effective basis, which also indicates the validity of the approximation in the quantum master equation.

\section{§3. Magnetization Process at $T=0$}

Figure ??(a) shows the result of the magnetization process for $\tilde{t}_{\max }=30000, \tilde{h}_{\max }=15$ and $\tilde{D}=0.01$ together with the complete stair case of the ground state of the model of $D=0$. Every step indicates the position of a level crossing at which the magnetization grows from $M$ to $M+1$. Because a final value of the magnetization 
reaches full saturation, the process is almost adiabatic at every crossing. Figure ??(b) shows $\mathrm{d} M / \mathrm{d} \tilde{h}$ for $\tilde{D}=0.01$. Peaks with various heights induced by the DM interaction are formed at the level-crossing positions denoted by crosses. It is noticable that the heights of the peaks are different from each other. Especially, the first peak is the highest. The heights gradually become small as the field is increased although the heights in a few last peaks get higher again. These features of the different peak heights agree well with the experimental results in $\mathrm{d} M / \mathrm{d} H$ for $\mathrm{Fe}_{6}$. 3 and $\mathrm{Fe}_{10}$ (4)

Single-ion-type anisotropy and dipole-dipole interactions may causes broadening of $\mathrm{d} M / \mathrm{d} H$. However, the contribution of the single-ion anisotropy is considered to be small because the orbital angular momentum vanishes in the each iron ion $\mathrm{Fe}^{3+}$ of the above materials. The offdiagonal element $\left\langle\phi_{2 M+1}\left|\mathcal{H}_{\text {dip }}\right| \phi_{2 M}\right\rangle$ of the dipole-dipole interactions,

$$
\mathcal{H}_{\mathrm{dip}} \equiv \sum_{i, j}\left(g \mu_{\mathrm{B}}\right)^{2}\left[\frac{\boldsymbol{S}_{i} \cdot \boldsymbol{S}_{j}}{r_{i j}^{3}}-\frac{3}{r_{i j}^{5}}\left(\boldsymbol{S}_{i} \cdot \boldsymbol{r}_{i j}\right)\left(\boldsymbol{S}_{j} \cdot \boldsymbol{r}_{i j}\right)\right]
$$

vanishes because $\left|\phi_{2 M}\right\rangle$ and $\left|\phi_{2 M+1}\right\rangle$ have wave numbers $\pi$ and 0 , respectively. This means that contribution of the dipole-dipole interactions is also small. Thus, we conclude that the transition due to the alternating DM interactions is the major origin of the peaks of $\mathrm{d} M / \mathrm{d} H$ of $\mathrm{Fe}_{6}$ and $\mathrm{Fe}_{10}$ with different heights.

On the contrary the $\mathrm{d} M / \mathrm{d} H$ curve of $\mathrm{Fe}_{12}$, which is an abbreviation of $\left[\mathrm{Fe}\left(\mathrm{OCH}_{3}\right)_{2}(\mathrm{dbm})\right]_{12} \frac{13}{13}$ reveals that the height of the first peak in $\mathrm{d} M / \mathrm{d} H$ is not the laroest and that the second one is larger than the first one.14 It was reported that the lattice structure of this material has a large deviation from the regular polygon, while in $\mathrm{Fe}_{6}$ and $\mathrm{Fe}_{10}$, the lattices are almost regular polygons. In order to take the deviation into account, we study a lattice with locally non-uniform magnetic interaction $\left\{J_{i}\right\}$. In our calculations, however, the first peak is still the highest in the system with various sets of $\left\{J_{i}\right\}$.

In the dimer material $\mathrm{Fe}_{2}$, the second peak is higher than the first one.9) The interactions in these compounds should be clarified in the future.

So far we studied the adiabatic behavior for slow sweeps, which probes the magnetization processes in the ground state. Next, let us consider the dynamical effect of the sweeping of the magnetic field in the case of a larger speed. The result for $\tilde{t}_{\max }=7500$ is shown in Fig. ??. The magnetization at a field where the saturation value is reached in Fig. ??(a) becomes smaller. This means that there are some level crossings at which nonadiabatic transitions occur. At $\tilde{h}=0.7$, the magnetization jumps up to $M=1$ in the adiabatic process. However, in Fig. ??(a), the step height is clearly lower, suggesting a nonadiabatic transition at the first level crossing. In the fields after the first crossing before the second one, the state is given approximately by a linear combination of $\left|\phi_{0}\right\rangle$ and $\left|\phi_{1}\right\rangle$, i.e., $|\psi\rangle=c_{0}\left|\phi_{0}\right\rangle+c_{1}\left|\phi_{1}\right\rangle$, where $\left|\phi_{0}\right\rangle$ and $\left|\phi_{1}\right\rangle$ behave almost as eigenstates for the field away from the crossings. The probabilities of $\left|\phi_{0}\right\rangle$ and $\left|\phi_{1}\right\rangle$ are found to be $\left|c_{0}\right|^{2}=0.22$ and $\left|c_{1}\right|^{2}=0.78$, respectively. The plateau height $m$ satisfies the relation $m=\left|c_{1}\right|^{2}=1-\left|c_{0}\right|^{2}=0.78$. For fields between the second and the higher-field crossings, the growth of the magnetization is briefly linear. The population of $\left|c_{1}\right|^{2}=0.78$ stays in the ground state, which gives the linear increase of the magnetization. The saturated magnetization for $\tilde{t}_{\max }=7500$ agrees with $15 \times m$. These facts indicate that the system shows almost adiabatic process at crossings other than the first one within this sweeping speed and that the energy gap at the first crossing is found to be the smallest. Thus, the transition at the first crossing plays a role of a bottleneck process for the growth of the magnetization. Note here that the population at $\left|\phi_{0}\right\rangle$ does not cause changes in the magnetization process within the present approximation although it is also exposed to nonadiabatic transitions in higher fields in the full-size Hamiltonian. To obtain such nonadiabatic transitions, an improvement of the method of calculation is required.

If the nonadiabatic transitions are experimentally observed as a quantum effect, we could capture the phenomena of the states which branch at the level crossings. Duration times $\tilde{t}_{\max }=7500$ and $\tilde{t}_{\max }=30000$ correspond to $3 \sim 6$ [ns] and 11 22 [ns] in real time, respectively, by using $J / k_{\mathrm{B}}=10 \sim 20[\mathrm{~K}]$ estimated from the susceptibility fit of $\mathrm{Fe}_{6}$ and $\mathrm{Fe}_{10}$. In the present experiments, on the other hand, the duration time of the pulsed field is in order of 10 [ms]. In order to find the nonadiabatic phenomena in $\mathrm{Fe}_{6}$ and $\mathrm{Fe}_{10}$, either a faster sweeping speed or a value of $\tilde{D}$ smaller than 0.01 is necessary. When $\tilde{D}$ is small, it is expected that $D$ is proportional to the energy gap at the level crossing. The amplitude of the gap determines whether the transition is adiabatic or nonadiabatic. Then, for the appearance of the nonadiabatic features $D / k_{\mathrm{B}}$ must be in order of $0.1[\mathrm{mK}]$, if the duration time is $10[\mathrm{~ms}]$ and $J / k_{\mathrm{B}}=10[\mathrm{~K}]$. An experimental estimate of the amplitude of the antisymmetric interactions in $\mathrm{Fe}_{6}$ and $\mathrm{Fe}_{10}$ would be very helpful.

\section{$\S 4$. Magnetization Process at Finite Tempera- tures}

In the previous subsection, we studied the dynamical magnetization process in the pure quantum-mechanical way. At finite temperatures, however, influence from the surrounding environment may have significant effects. Here, we study the effect of the thermal environment on the iron clusters.

Figure ?? shows the result of the magnetization process for the case of $\tilde{D}=0.01, \lambda=0.005, \tilde{h}_{\max }=12$ and $\tilde{t}_{\max }=60000$ in the condition of temperature $k_{\mathrm{B}} T / J=0.05$. A satellite peak in $\mathrm{d} M / \mathrm{d} \tilde{h}$, is clearly observed around $\tilde{h}=0.9$ in the receding side of the field near the main peak at $\tilde{h}=0.7$. This satellite peak corresponds to a dent of the magnetization process at an edge of the magnetization step. This satellite peak is considered to be a consequence of the magnetic Föhn effect.11) The key mechanism of the magnetic Föhn effect is the difference between two kinds of speed characterizing properties of the total system. One is a speed determined by the LZS mechanism, below which the quantum transition is almost adiabatic. The other is a speed controlled by $\mathcal{H}_{\text {int }}$, above which the supply of energy from 
the heat bath is not fast enough to keep the system in the equilibrium during the change of the field. If the sweeping speed of the field is between these two, the magnetic Föhn effect occurs. This effect has been proposed as a general phenomenon in the processes which are almost adiabatic but with a little inflow of heat.11) Because the process is almost adiabatic, the system is almost in the ground state, that is in the lower-energy state. However, due to the interaction with the environments, some population is excited to the higher-energy level. But it is not enough to realize the thermal equilibrium distribution. This excitation occurs as far as the equilibrium population at the higher-energy level $\rho_{\text {eq }}^{(2)}\left(\propto \mathrm{e}^{-\beta \Delta(\tilde{h})}\right)$ is larger than the dynamical one of the system $\rho^{(2)}(t)$, when $\Delta(\tilde{h})$ is the energy difference between the states. When the field gets away from the crossing point, the energy difference increases; $\rho_{\text {eq }}^{(2)}$ decreases rapidly and becomes smaller than $\rho^{(2)}(t)$. There, the population at higherenergy level begins to relax to the lower-energy level. In this way, $\rho^{(2)}(t)$ has a maximum at a field $\tilde{h}$ in the receding side from the crossing point. The behavior of $\rho^{(2)}(t)$ causes a dent of the magnetization process.

In the receding side of the field near the main peak at $\tilde{h}=1.4$, a smaller swell appears around $\tilde{h}=1.6$. In this case, the satellite due to the thermal redistribution is being merged into the main peak. Situations of merging depend on the distance between the satellite and the center of the main peak, and on the width of the main peak. The former is determined by the details of the heat bath and those of the interface interaction. The latter is given quantum-mechanically by the energy gap at the avoided level crossing. In the present case, the merging behavior is induced because the width of this main peak becomes larger in higher fields. This effect causes a gentle slope in the receding side compared with that in the approaching side. Thus, the asymmetry of the experimental peak in the field derivative of the magnetization is understood as a consequence of the magnetic Föhn effect induced by the thermal environment.

Let us consider the temperature dependence of the satellite peak. Results are shown in Fig. ??. As the temperature is decreased, the satellite peak gets smaller and almost vanishes at $k_{\mathrm{B}} T / J=0.01$. It is characteristic that the satellite peak disappears without merging with the main peak. This temperature corresponds to $0.09 \sim 0.18 \mathrm{~K}$ if we take a experimental estimation of $J / k_{\mathrm{B}}$ to be $10 \sim 20 \mathrm{~K}$. This means that in the experiments for $\mathrm{Fe}_{6}$ at such low temperatures, the satellite peaks in $\mathrm{d} M / \mathrm{d} H$ would disappear in the way we observed. A detailed comparison with such an experiment is required.

\section{$\S 5$. Concluding Remarks}

We have studied properties and the origin of the quantum transitions observed in the magnetization process of the nanoscale iron clusters at $T=0$ and at finite temperatures. We have proposed an approximate procedure to make the numerical analysis possible and obtain the magnetization process for the system where the dimension of the basis states is large and conventional method is hardly applicable. We have found that the antisymmetric interaction reproduces the characteristics of the heights of the peaks in $\mathrm{d} M / \mathrm{d} H$ at $T=0$. The effect of nonadiabatic magnetization process was also discussed. The first level crossing plays a role of the major branching point. At finite temperatures, the asymmetric behavior of the peaks in $\mathrm{d} M / \mathrm{d} H$ is induced by the thermal environment influencing the spin system as the magnetic Föhn effect. In experiments, observation of the magnetization process is a powerful and direct method to estimate the coupling amplitude $J$ of the Heisenberglike exchange interactions. However, the magnetization process yeilds not only information on $J$ but also other information characterizing the properties of the materials. In this paper, we have clarified such aspects of the iron clusters.

\section{Acknowledgements}

We would like to thank K. Saito for valuable discussions. We also thank Y. Narumi, Y. Ajiro, Y. Inagaki and $\mathrm{K}$. Kindo for providing us with the experimental data before publication and for stimulating discussions. We wish to thank H. De Raedt for his kind critical reading of the manuscript. This work is supported partly by a Grant-in-Aid for Scientific Research from the Ministry of Education, Science, Sports and Culture, Japan. One of the authors (H.N.) is supported by Research Fellowships of the Japan Society for the Promotion of Science for Young Scientists. A part of the computations was performed using the facilities of the Supercomputer Center, Institute for Solid State Physics, University of Tokyo.

1) A. Caneschi, D. Gatteschi, C. Sangregorio, R. Sessoli, L. Sorace, A. Cornia, M. A. Novak, C. Paulsen and W. Wernsdorfer: J. Magn. Magn. Mater. 200 (1999) 182 and references therein.

2) Experimentally, the magnetization is obtained from the integration of observed $\mathrm{d} M / \mathrm{d} H$.

3) A. Caneschi, A. Cornia, C. Fabretti, S. Foner, D. Gatteschi, R. Grandi and L. Schenetti: Chem. Eur. J. 2 (1996) 1379.

4) K. L. Taft, C. D. Delfs, G. C. Papaefthymiou, S. Foner, D. Gatteschi and J. Lippard: J. Am. Chem. Soc. 116 (1994) 823.

5) L. Landau: Phys. Z. Sowjetunion 2 (1932) 46.

6) C. Zener: Proc. R. Soc. London Ser. A 137 (1932) 696.

7) E. C. G. Stükelberg: Helv. Phys. Acta 5 (1932) 369.

8) S. Miyashita: J. Phys. Soc. Jpn. 64 (1995) 3207.

9) Y. Shapira, M. T. Liu, S. Foner, C. E. Dubé and P. J. Bonitatebus Jr.: Phys. Rev. B 59 (1999) 1046.

10) I. Chiorescu, W. Wernsdorfer, A. Müller, H. Bögge and B. Barbara: Phys. Rev. Lett. 84 (2000) 3454

11) K. Saito and S. Miyashita: cond-mat/0004027.

12) K. Saito, S. Takesue and S. Miyashita: Phys. Rev. B 61 (2000) 2397.

13) A. Caneschi, A. Cornia, A. C. Fabretti and D. Gatteschi: Angew. Chem. Int. Ed. Eng. 38 (1999) 1295.

14) Y. Ajiro and Y. Inagaki: private communications, Y. Narumi and K. Kindo: private communications. 
This figure "fig123.gif" is available in "gif" format from: http://arxiv.org/ps/cond-mat/0105227v1 
This figure "fig456.gif" is available in "gif" format from: http://arxiv.org/ps/cond-mat/0105227v1 